Review

\title{
Occurrence and Prevalence of Insect Pathogens in Populations of the Codling Moth, Cydia pomonella L.: A Long-Term Diagnostic Survey
}

\author{
Gisbert Zimmermann ${ }^{\dagger}$, Alois M. Huger ${ }^{\dagger}$ and Regina G. Kleespies * \\ Julius Kühn-Institute (JKI), Federal Research Centre for Cultivated Plants, \\ Institute for Biological Control, Heinrichstrasse 243, Darmstadt D-64287, Germany; \\ E-Mails: gisbert.zimmermann@gmx.net (G.Z.); a.huger@web.de (A.M.H.) \\ $\uparrow$ These authors are already retired. \\ * Author to whom correspondence should be addressed; E-Mail: regina.kleespies@jki.bund.de; \\ Tel.: +49-6151-407-226; Fax: +49-6151-407-290.
}

Received: 14 May 2013; in revised form: 15 July 2013 / Accepted: 16 July 2013 /

Published: 2 August 2013

\begin{abstract}
About 20,550 larvae, pupae and adults of the codling moth, Cydia pomonella L., were diagnosed for pathogens during long-term investigations (1955-2012) at the Institute for Biological Control in Darmstadt, Germany. The prevailing entomopathogens diagnosed in these studies were insect pathogenic fungi, especially Beauveria bassiana and Isaria farinosa, the microsporidium, Nosema carpocapsae, the Cydia pomonella granulovirus $(\mathrm{CpGV})$, as well as mostly undetermined bacteria. While the $\mathrm{CpGV}$ was observed exclusively in larvae and pupae from laboratory colonies or from field experiments with this virus, entomopathogenic fungi were most frequently diagnosed in last instars in autumn and in diapausing larvae and pupae in spring. B. bassiana was identified as the major fungal pathogen, causing larval prevalences of $0.9 \%$ to $100 \%$ (mean, about $32 \%$ ). During prognostic long-term studies in larvae and adults of $C$. pomonella, $N$. carpocapsae was diagnosed in codling moth populations from various locations in Germany. The mean prevalence generally ranged between $20 \%$ and 50\%. Experiments revealed that the fecundity and fertility of microsporidia-infected female adults were significantly reduced compared to healthy ones. The results underpin the importance of naturally occurring microbial antagonists and represent a base for further ecological studies on developing new or additional biological and integrated control strategies.
\end{abstract}


Keywords: Cydia pomonella; diagnosis; insect pathogens; granulovirus; fungi; Beauveria bassiana; Nosema carpocapsae; host-pathogen-interactions

\section{Introduction}

The codling moth, Cydia pomonella L. (Lepidoptera, Tortricidae), is a major and nearly worldwide occurring pest insect, mainly of apple trees, necessitating regular control measures. Generally, chemical insecticides are used, but also biological control methods, like pheromone mating disruption, as well as the specific $C$. pomonella granulovirus (CpGV), are applied [1-6]. Concerns on safety and the environmental impact of insecticides, as well as the development of resistance have led to an increased use of CpGV. However, recently, some codling moth populations also showed a reduced susceptibility against $\mathrm{CpGV}$ products, stimulating the search for further biological control measures [7-16].

Several naturally occurring microbial antagonists have been reported from C. pomonella, including viruses, bacteria, fungi, microsporidia and nematodes [12,17-20]. So far, CpGV, the bacterium, Bacillus thuringiensis, the fungus, Beauveria bassiana, the microsporidium, Nosema carpocapsae, and, recently, also, nematodes of the genus, Steinernema, have been tested in the lab or in the field $[7,8,12,15,21-29]$.

Although the codling moth is a key pest in apple orchards, only a few ecological investigations on naturally occurring antagonists, especially insect pathogenic microorganisms, and on their importance as mortality factors in codling moth populations in Germany or Europe were conducted [7,18,25,30-34]. In this context, only a few studies on the causes of mortalities of diapausing larvae during hibernation are available, whereby pathogens could play an important role [34,35]. For example, Zelger et al. [36] found a winter mortality of $20-37 \%$, mainly caused by weather conditions and general biotic factors, which are not clearly defined.

Recently, a review and a database on entomopathogens of insects and other arthropods found during long-term diagnostic studies at the Institute for Biological Control, Darmstadt, were published [37,38]. The data reported herein summarizes the type and frequency of pathogens found in 20,550 living, diseased or dead codling moth individuals that were diagnostically examined and the entomopathogens determined (E. Müller-Kögler, fungi: 1955-1974; A. Krieg, bacteria, rickettsiae, viruses: 1955-1989; A.M. Huger, viruses, bacteria, rickettsiae, microsporidia, protists: 1957-1991; G. Zimmermann, fungi: 1974-2005; R.G. Kleespies, viruses, bacteria, rickettsiae, microsporidia, protists: since 1991). As most of the results of these studies are not published and only few of them are briefly outlined in German annual reports of the former Federal Biological Research Centre for Agriculture and Forestry (BBA), a detailed presentation on C. pomonella is given here. We believe that these results gained over a long period of time will stimulate further ecological and pathological studies on $C$. pomonella, in order to enrich the understanding of the efficacy of natural microbial control factors within the population development. They also may initiate further approaches to preserve natural ecosystems and to develop new strategies within an integrated control system. 


\section{Material and Methods}

\subsection{Examination and Identification}

For diagnostic studies and the identification of entomopathogenic and other microorganisms found in all stages of the codling moth, different microbiological and histopathological methods and techniques were used [22,39-50]. First, all specimens were examined microscopically (stereo and light microscope) and, if necessary, also by transmission electron microscope (TEM). In case of an infection by fungi, the larvae and pupae were surface-sterilized with $0.1 \%$ hypochlorite and, then, transferred to Petri dishes containing a moist filter paper or a moist cotton plug to stimulate outgrowth of the fungus from the cadaver. For identification, bacteria and fungi were isolated on various media, e.g., Tryptic soy broth (TSB + 1.5\% agar; Otto Norwald KG, Hamburg, Germany) or malt peptone agar (3\% malt extract, $0.5 \%$ peptone, $1.5 \%$ agar), respectively, with or without antibiotics (chloramphenicol $50 \mathrm{mg} / \mathrm{L}$ ). In most cases, a clear identification of bacteria was not conducted. As bacteria are members of the natural gut flora of insects, they can easily be found in dead individuals of $C$. pomonella, even if these had died because of age-related reasons or due to abiotic or other biotic reasons. Fungi were identified according to [51-54]. Fungal names were adapted, as far as possible, to the actual nomenclature, e.g., the new name of Paecilomyces farinosus is Isaria farinosa [55,56]. So-called wound or secondary pathogens, including saprophytic fungi, such as Aspergillus, Penicillium and some Zygomycetes, were only identified to the genus level. As these fungi often are in the gut or on the cuticle of dead insects, their role as insect pathogens for the majority is seldom clear.

To verify $\mathrm{CpGV}$, generally, a fast and simple routine procedure was employed: If wet-mount preparations of fat body tissue strikingly revealed many or even myriads of tiny particles in rapid Brownian movement in phase contrast and dark field, small drops of this material were applied onto Formvar-coated grids, negatively stained by $2.0 \%$ phosphotungstic acid and analyzed in TEM-studies. For cyto- and histo-pathological investigations, stained serial sections were employed in light microscope and TEM (see below).

For identifying microsporidia, morphological features of spores and lifecycle stages proved useful when examining squash preparations of host tissues and Giemsa-stained smears. To clarify histo- and cyto-pathological events, stained serial sections of tissues or entire specimens were necessary using the light and TE-microscope. For light microscopy, individuals were fixed with Duboscq-Brazil's alcoholic Bouin's and embedded in Histosec (Merck, Darmstadt, Germany). Serial sections at 6-9 $\mu \mathrm{m}$ were stained with Heidenhain's iron hematoxylin, counterstained with erythrosin and examined in a Leica photomicroscope, model DMRB (Leica, Bensheim, Germany). For electron microscope studies of ultrathin sections, larval tissues were fixed overnight at $4{ }^{\circ} \mathrm{C}$ in $3 \%$ glutaraldehyde in Veronal buffer (pH 7.2) and postfixed in 1-2\% osmium tetroxide in the same buffer for 6-20 hours. After dehydration in ascending ethanol series, tissues were embedded in a 7:3 mixture of butyl and $n$-methymethacrylate [43]. Thin sections, double-stained with uranyl acetate and lead citrate, were examined in a Zeiss EM 9 and a Zeiss EM 902 (since 1991) (Zeiss, Oberkochen, Germany).

The long-term studies on the occurrence and prevalence of $N$. carpocapsae were conducted exclusively by Huger between 1972 and 1978 for larvae and from 1972 until 1990 for the adults $[33,57,58]$. The specimens were collected in commercial orchards by Mr. Zotzmann from the 
former Plant Protection Office in Frankfurt/Main using light traps, pheromone traps or from hibernation cages (wire gauze cages fixed on corrugated cardboard bands around apple tree stems to prevent predation e.g. by birds). As genetic investigations have documented that microsporidia belong to fungi (e.g., $[59,60])$, they are mentioned here as Fungus/Microsporidium.

\subsection{Experiments with Nosema carpocapsae}

The effect of the microsporidium N. carpocapsae on the fecundity and fertility of C. pomonella was examined in six laboratory experiments and, so far, only briefly published [25]. Ten female and 20 male adults, deriving from a healthy and a microsporidia-infected laboratory-reared colony were used in each bioassay. Ten groups of one female and 2 males were kept in a plastic box $(\varnothing, 6 \mathrm{~cm}$, height, $5 \mathrm{~cm}$ ) that was lined inside with a thin layer of foamy material in order to concentrate egg-laying onto the clean, smooth and transparent lid. The boxes were stored in an incubator at $23{ }^{\circ} \mathrm{C}$ and permanent light sources on each side. The number of eggs laid per female and the number of hatched larvae were determined. For statistical analysis of the data on fecundity and fertility, the $S A S$ System for Windows, Version 9.1 was used. Significant differences between the means were calculated using the glm-procedure (general linear models) and the Student-Newman-Keuls-test $(p \leq 0.05)$ and angular transformed data (fertility).

\section{Results}

\subsection{General Information on Accessions}

Altogether, 89 accessions of living and/or dead larvae, pupae and adults of C. pomonella mostly from field populations in commercial orchards were examined. They included about 20,550 individuals, i.e., 8,600 larvae, 800 pupae, 10,200 adults, as well as a mixture of larvae and pupae $(\mathrm{n}=900)$ and of all life stages $(n=50)$ that could not be separated clearly. About $90 \%(n=79)$ of all accessions were from Germany, one each from Italy and Israel, two from Austria and six from Switzerland. Accessions were mainly sent by plant protection services, some of them also by companies maintaining a codling moth colony. Most larvae were last instars or diapausing larvae collected in autumn or spring, respectively, from corrugated cardboard bands. Adults were caught in light traps or in pheromone traps or were collected from hibernation cages and examined for microsporidia and other pathogens during prognostic studies (51 accessions, 57.3\%). Furthermore, 25 accessions (28.1\%) contained larvae, pupae and adults of codling moth laboratory colonies.

\subsection{Pathogens, Other Microorganisms and Nematodes of C. pomonella (Larvae, Pupae, Adults) from}

\section{Field Populations}

In specimens received from field populations, insect pathogenic and other fungi represented the major group of diagnosed organisms, followed by bacteria, microsporidia (mostly N. carpocapsae), the granulovirus, $\mathrm{CpGV}$, and nematodes (Table 1 and Figure 1). Fungi were mostly diagnosed in last instars and in diapausing larvae in autumn and spring, respectively, and often also mixed infections of two or three species were observed. The predominant entomopathogenic species was $B$. bassiana, followed by I. farinosa (syn. P. farinosus), Hirsutella spp., Lecanicillium spp. (syn. Verticillium lecanii), 
Isaria fumosorosea (syn. Paecilomyces fumosoroseus) and Metarhizium anisopliae. B. bassiana and I. farinosa were also detected frequently in mixed infections, and in some cases, they were hyperparasitized by the ascomycete, Syspastospora parasitica (syn. Melanospora parasitica) [21,61]. Other genera, such as Alternaria, Aspergillus, Cephalosporium, Cladosporium, Fusarium, Mucor or Penicillium, also occurred relatively often. Whether these fungi are true entomopathogens is not yet clear. Bacteria were mostly diagnosed in dead specimens of all developmental stages of C. pomonella. Unidentified spore-formers and Serratia spp. were predominant. The microsporidium, N. carpocapsae, was found in larvae and pupae, as well as in living and dead adults, where it was diagnosed as the only pathogen. Occasionally, a double infection with the $\mathrm{CpGV}$, some fungi, bacteria and unidentified nematodes was observed. CpGV was detected exclusively in dead larvae and pupae.

Table 1. Overview on diagnosed pathogens, other microorganisms and nematodes in C. pomonella (larvae, pupae, adults) detected in the Institute for Biological Control (1955-2012); (modified from [37]; AT, Austria; CH, Switzerland; DE, Germany).

\begin{tabular}{|c|c|c|}
\hline Pathogen-Group & Pathogens/Microorganisms/Nematodes & Origin (country) \\
\hline \multirow[t]{2}{*}{ Viruses } & Granulovirus & $\mathrm{CH}, \mathrm{DE}$ \\
\hline & Granulovirus + Nosema carpocapsae & $\mathrm{CH}, \mathrm{DE}$ \\
\hline \multirow[t]{7}{*}{ Bacteria } & Bacteria, unidentified & $\mathrm{DE}$ \\
\hline & Bacteria (spore-formers), unidentified & $\mathrm{DE}$ \\
\hline & Serratia liquefaciens & $\mathrm{DE}$ \\
\hline & Serratia sp. & $\mathrm{DE}$ \\
\hline & Hafnia alvei + Serratia sp. + Pseudomonas sp. & $\mathrm{DE}$ \\
\hline & Serratia sp. + Nosema carpocapsae & $\mathrm{DE}$ \\
\hline & Bacillus cereus + nematodes, unidentified & $\mathrm{DE}$ \\
\hline \multirow[t]{17}{*}{ Fungi } & Alternaria sp. & $\mathrm{DE}$ \\
\hline & Aspergillus flavus & $\mathrm{DE}$ \\
\hline & Aspergillus sp. & $\mathrm{AT}, \mathrm{DE}$ \\
\hline & Beauveria bassiana & AT, DE \\
\hline & $\begin{array}{l}\text { Beauveria bassiana, hyperparasitized by Syspastospora parasitica } \\
\text { (Melanospora parasitica) }\end{array}$ & $\mathrm{DE}$ \\
\hline & Beauveria sp. & AT \\
\hline & Cephalosporium sp. & $\mathrm{AT}, \mathrm{DE}$ \\
\hline & Cladosporium sp. & AT \\
\hline & Fusarium avenaceum & AT \\
\hline & Fusarium sp. & $\mathrm{AT}, \mathrm{DE}$ \\
\hline & Hirsutella gigantea & AT \\
\hline & Hirsutella sp. & $\mathrm{AT}, \mathrm{CH}, \mathrm{DE}$ \\
\hline & Hirsutella subulata & AT \\
\hline & Isaria farinosa & $\mathrm{AT}, \mathrm{DE}$ \\
\hline & $\begin{array}{l}\text { Isaria farinosa hyperparasitized by Syspastospora parasitica } \\
\text { (Melanospora parasitica) }\end{array}$ & $\mathrm{AT}, \mathrm{DE}$ \\
\hline & Isaria fumosorosea & $\mathrm{DE}$ \\
\hline & Lecanicillium sp. (Verticillium lecanii) & $\mathrm{DE}$ \\
\hline
\end{tabular}


Table 1. Cont.

\begin{tabular}{|c|c|c|}
\hline Pathogen-Group & Pathogens/Microorganisms/Nematodes & Origin (country) \\
\hline & Metarhizium anisopliae & AT \\
\hline & Mucor sp. & $\mathrm{AT}, \mathrm{DE}$ \\
\hline & Penicillium sp. & $\mathrm{AT}, \mathrm{DE}$ \\
\hline & Verticillium sp. & $\mathrm{DE}$ \\
\hline & Mixed infections & \\
\hline & Alternaria sp. + Cephalosporium sp. & $\mathrm{DE}$ \\
\hline & Alternaria sp. + Fusarium sp. & $\mathrm{DE}$ \\
\hline & Aspergillus sp. + Fusarium sp. & $\mathrm{AT}, \mathrm{DE}$ \\
\hline & Aspergillus sp. + Mucor $\mathrm{sp}$. & AT \\
\hline & Aspergillus sp. + Fusarium sp. + Penicillium $\mathrm{sp}$. & AT \\
\hline & Beauveria bassiana + bacteria, unidentified & AT \\
\hline & Beauveria bassiana + Aspergillus sp. & AT \\
\hline & Beauveria bassiana + Cephalosporium $\mathrm{sp}$. & $\mathrm{DE}$ \\
\hline & Beauveria bassiana + Cladosporium sp. & AT \\
\hline & Beauveria bassiana + Hirsutella sp. & $\mathrm{DE}$ \\
\hline & Beauveria bassiana + Mucor $\mathrm{sp}$ & $\mathrm{AT}$ \\
\hline & Beauveria bassiana + Isaria farinosa & $\mathrm{DE}$ \\
\hline & Beauveria bassiana + Penicillium $\mathrm{sp}$ & $\mathrm{DE}$ \\
\hline & Beauveria bassiana + Aspergillus sp. + Hirsutella $\mathrm{sp}$. & AT \\
\hline & Beauveria bassiana + Mucor $\mathrm{sp} .+$ Penicillium $\mathrm{sp}$. & AT \\
\hline & Beauveria bassiana + Penicillium sp. + Aspergillus sp. & AT \\
\hline & Hirsutella sp. + Aspergillus sp. & AT \\
\hline & Hirsutella sp. + Mucor sp. & AT \\
\hline & Isaria farinosa + Alternaria sp. & $\mathrm{DE}$ \\
\hline & Isaria farinosa + Fusarium sp. & $\mathrm{DE}$ \\
\hline & Isaria farinosa + Penicillium $\mathrm{sp}$ & AT \\
\hline & Isaria farinosa + Beauveria bassiana + Penicillium $\mathrm{sp}$. & AT \\
\hline & Isaria farinosa + nematodes, unidentified & $\mathrm{DE}$ \\
\hline & Paecilomyces sp. + Alternaria sp. & $\mathrm{DE}$ \\
\hline & Paecilomyces sp. + Beauveria sp. & $\mathrm{DE}$ \\
\hline & Paecilomyces sp. + Mucor sp. & $\mathrm{DE}$ \\
\hline & Paecilomyces sp. + Verticillium sp. & $\mathrm{DE}$ \\
\hline & Penicillium sp. + Alternaria sp. & $\mathrm{DE}$ \\
\hline & Penicillium sp. + Aspergillus $\mathrm{sp}$. & $\mathrm{DE}$ \\
\hline & Penicillium sp. + Cephalosporium sp. & $\mathrm{DE}$ \\
\hline & Penicillium sp. + Mucor sp. & $\mathrm{AT}, \mathrm{DE}$ \\
\hline & Verticillium sp. + Alternaria sp. & $\mathrm{DE}$ \\
\hline & Verticillium sp. + Penicillium $\mathrm{sp}$. & $\mathrm{DE}$ \\
\hline \multirow[t]{4}{*}{ Fungi/Microsporidia } & Nosema carpocapsae & $\mathrm{CH}, \mathrm{DE}$ \\
\hline & Nosema carpocapsae + nematodes, unidentified & $\mathrm{DE}$ \\
\hline & Nosema carpocapsae + fungi, unidentified & $\mathrm{DE}$ \\
\hline & Microsporidia, unidentified + bacteria, unidentified & $\mathrm{CH}$ \\
\hline Nematodes & Nematodes, unidentified & $\mathrm{DE}$ \\
\hline
\end{tabular}


Figure 1. Important entomopathogens of the codling moth, Cydia pomonella. (A) Thin section of granulovirus ( $\mathrm{CpGV}$ ) with capsule-shaped virus occlusion bodies $(\mathrm{C})$, formed by proteinaceous granulin $(\mathrm{G})$. Each virus capsule $(\mathrm{C})$ harbors one rod-shaped virion $(\mathrm{V})$. Longitudinal sections $(\mathrm{Cl})$ and cross sections $(\mathrm{Cc})$ of virus capsules (transmission electron microscope); (B) Different morphologies of the entomopathogenic fungus, Beauveria bassiana, on three codling moth larvae (stereomicroscope); (C) Spores (S) of the microsporidium, Nosema carpocapsae (phase contrast microscope); (D) Longitudinal section of larval midgut epithelium (M) with heavy infection by N. carpocapsae. Rounded batches of the dark colored spores (S) are expelled from the cylindrical epithelium cells into the midgut lumen $(\mathrm{L})$. Mu, muscularis of the midgut. (Bright field microscope, staining: Hematoxylin Heidenhain-Erythrosin).
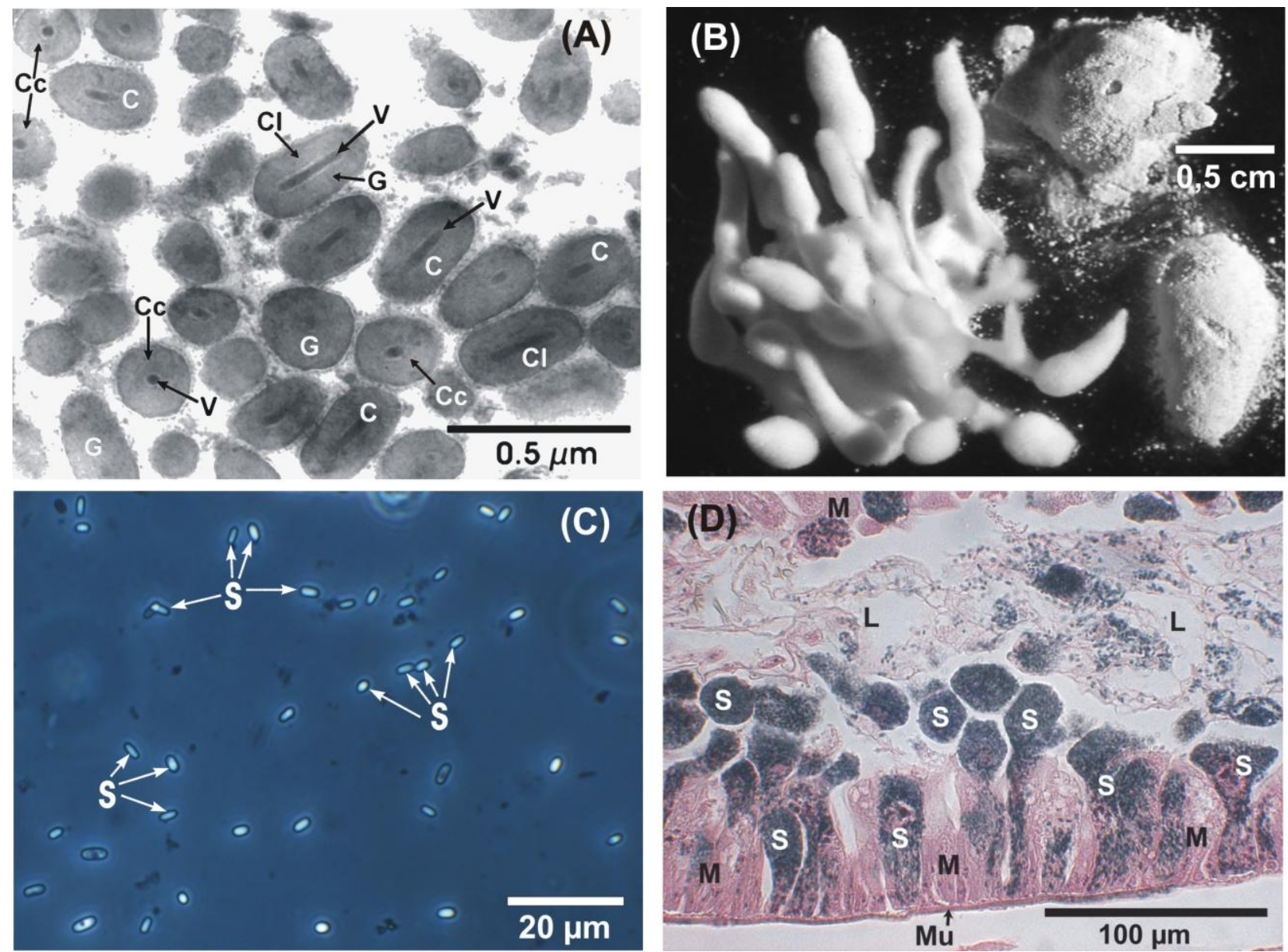

\subsection{Pathogens and Other Microorganisms of C. pomonella (Larvae, Pupae, Adults) from}

\section{Laboratory Colonies}

In $28 \%$ of all accessions containing larvae, pupae and adults from laboratory colonies of C. pomonella, mostly infections by $\mathrm{CpGV}$ and $N$. carpocapsae, as well as by bacteria of the genera, Serratia and Pseudomonas, were identified, besides undetermined bacteria (Table 2). In one accession, B. bassiana and I. farinosa were diagnosed in larvae. It is assumed that these larvae already were infected when brought to the laboratory. 
Table 2. Diagnosed pathogens and other microorganisms in C. pomonella (larvae, pupae, adults), from laboratory colonies.

\begin{tabular}{|c|l|c|}
\hline Pathogen-Group & \multicolumn{1}{|c|}{ Pathogens/Microorganisms } & Accessions (n) \\
\hline \multirow{5}{*}{ Viruses } & Granulovirus & 4 \\
\cline { 2 - 3 } & Granulovirus + Nosema carpocapsae & 1 \\
\cline { 2 - 3 } & Granulovirus + microsporidia, unidentified & 3 \\
\hline \multirow{5}{*}{ Bacteria } & Bacteria, unidentified & 4 \\
\cline { 2 - 3 } & Serratia liquefaciens & 1 \\
\cline { 2 - 3 } & Bacteria, unidentified+ Serratia sp. & 1 \\
\cline { 2 - 3 } & $\begin{array}{l}\text { Bacteria, unidentified + microsporidia, } \\
\text { unidentified }\end{array}$ & 1 \\
\cline { 2 - 3 } & Serratia sp. + Pseudomonas sp. & 1 \\
\hline \multirow{2}{*}{ Fungi } & Beauveria bassiana & 6 \\
\cline { 2 - 3 } & Isaria farinosa & 1 \\
\hline \multirow{2}{*}{ Fungi/Microsporidia } & Nosema carpocapsae & 1 \\
\cline { 2 - 3 } & Nosema carpocapsae + Serratia sp. & 1 \\
\hline
\end{tabular}

\subsection{Occurrence and Prevalence of the Most Frequent Pathogens}

The most frequent entomopathogens found in larvae, pupae or adults of all examined $C$. pomonella were the CpGV (10.1\%), B. bassiana (23.6\%) and $N$. carpocapsae (57.3\%). Therefore, some special aspects on their occurrence and prevalence in $C$. pomonella are presented here.

\subsubsection{Cydia pomonella Granulovirus (CpGV)}

CpGV was exclusively found in larvae and pupae from apparently inadvertently contaminated laboratory colonies or from field experiments, where the $\mathrm{CpGV}$ was applied before as plant protection measure (infestation rates 1.0-100\%). Thus, no evidence for a natural occurrence of $\mathrm{CpGV}$ was obtained during our studies.

\subsubsection{Beauveria bassiana}

In nearly all specimens of diseased or dead last instars in autumn or in diapausing larvae, as well as in pupae in spring, the fungus, B. bassiana, was detected during the whole period of diagnostic survey in various populations and different areas in Germany and one in Austria (Table 3). The number of individuals per accession was between three and 2,355. This documents that B. bassiana is the most important entomopathogenic fungus of $C$. pomonella under natural conditions irrespective of the year or origin. As mentioned, mixed infections with other entomopathogenic fungi, mainly I. farinosa and Hirsutella sp., as well as with other species were observed. In all accessions with specimens being only infected by B. bassiana, the prevalence of examined larvae and pupae ranged between $0.9 \%$ and $100 \%$; on average, about $32 \%$. The rate of mixed infections with other fungi was $0.3 \%$ to $5.4 \%$; in single cases, up to $25 \%$. 
Table 3. Occurrence and prevalence of the entomopathogenic fungus, Beauveria bassiana (B.ba.), in C. pomonella (larvae and pupae; the origin mostly refers to the address of the sender and not always to the location of the codling moth).

\begin{tabular}{|c|c|c|c|}
\hline Origin & Year & Results & $\begin{array}{l}\text { Infestation in \% and total number } \\
\text { of individuals examined (n) }\end{array}$ \\
\hline \multicolumn{4}{|l|}{ Austria } \\
\hline \multirow[t]{10}{*}{ Kronberg } & \multirow[t]{9}{*}{1971} & B.ba. & 36.9 (111) \\
\hline & & B.ba. + Aspergillus sp. & 1.8 \\
\hline & & B.ba. + Aspergillus sp. + Hirsutella $\mathrm{sp}$. & 0.9 \\
\hline & & B.ba. + bacteria, unidentified & 0.9 \\
\hline & & B.ba. + Cladosporium sp. & 1.8 \\
\hline & & B.ba. + I. farinosa + Penicillium sp. & 1.8 \\
\hline & & B.ba. + Mucor sp. & 1.8 \\
\hline & & B.ba. + Mucor sp. + Penicillium sp. & 0.9 \\
\hline & & B.ba. + Penicillium sp. + Aspergillus sp. & 1.8 \\
\hline & 1972 & B.ba. & $\mathbf{5 9 . 9}(2,355)$ \\
\hline \multicolumn{4}{|l|}{ Germany } \\
\hline \multirow[t]{2}{*}{ Dossenheim } & \multirow[t]{2}{*}{1983} & B.ba. & $\mathbf{0 . 9}(1,954)$ \\
\hline & & B.ba. + Paecilomyces sp. & 0.3 \\
\hline \multirow[t]{9}{*}{ Frankfurt } & 1955 & B.ba. & $88.2(17)$ \\
\hline & \multirow[t]{3}{*}{1973} & B.ba. & $87.5(24)$ \\
\hline & & B.ba. + I. farinosa & 4.2 \\
\hline & & B.ba. + Penicillium sp. & 4.2 \\
\hline & \multirow[t]{2}{*}{1974} & B.ba. & $87.8(74)$ \\
\hline & & B.ba. + Syspastospora parasitica & 5.4 \\
\hline & \multirow[t]{3}{*}{1975} & B.ba. & \multirow[t]{3}{*}{-} \\
\hline & & B.ba. + Cephalosporium sp. & \\
\hline & & B.ba. + I. farinosa & \\
\hline Heidelberg & 1957 & B.ba. & $42.8(14)$ \\
\hline Mainz & 1967 & B.ba. & - \\
\hline \multirow[t]{3}{*}{ Neustadt/Meckenheim } & $1955 / 1$ & B.ba. & ca. 20 (ca. 25) \\
\hline & \multirow[t]{2}{*}{$1955 / 2$} & B.ba. & ca. 75 (ca. 52) \\
\hline & & Mucor sp. on B.ba. & ca. 25 \\
\hline Neustadt/Weisenheim & 1955 & B.ba. & $53.3(75)$ \\
\hline Offenbach & 1973 & B.ba. & $\mathbf{1 0 0}(23)$ \\
\hline \multirow[t]{8}{*}{ Stuttgart } & 1957 & B.ba. & $29.4(17)$ \\
\hline & 1973 & B.ba. & $8.2(49)$ \\
\hline & \multirow[t]{2}{*}{1975} & B.ba. & 30.0 (40) \\
\hline & & B.ba. I. farinosa & 2.5 \\
\hline & \multirow[t]{2}{*}{2002} & B.ba. & $19.2(52)$ \\
\hline & & B.ba. + Hirsutella $\mathrm{sp}$. & 1.9 \\
\hline & 2004 & B.ba. & $9.2(488)$ \\
\hline & 2005 & B.ba. & $2.4(454)$ \\
\hline Wiesbaden & 1958 & B.ba. & $100(5)$ \\
\hline
\end{tabular}




\subsubsection{Nosema carpocapsae and Its Effects on Fecundity and Fertility of C. pomonella}

In more than $50 \%$ of all examined accessions, the microsporidium, $N$. carpocapsae, was identified. Most of these accessions (39.1\%) were received within a prognostic survey on the prevalence of $N$. carpocapsae in common field populations of the codling moth. This pathogen was mainly found in last instars in autumn, as well as in adults caught in the field by light traps pheromone traps or collected from hibernation cages. N. carpocapsae may also occur in mixed infections with CpGV, unidentified bacteria or nematodes in laboratory colonies of the codling moth (see Tables 1 and 2). Larvae and adults from field populations originated from Hessen (Hassia) (Darmstadt, Frankfurt, Kriftel, Niederhofheim and Geisenheim), from Bayern (Bavaria) (Deutenkofen, Erlabrunn, Neuhaus, Igensdorf, Eichelsdorf, Pittersberg, Mallersdorf, Uffenheim and Weihenstephan) and from Baden-Württemberg (Baden-Wurttemberg) (Dossenheim, Heidelberg, Ludwigsburg and Karlsruhe Augustenberg). The diagnosed infestation rates of larvae (seven years, 1972-1978) and adults (19 years, 1972-1990) from various locations and regions are presented in Tables 4 and 5, respectively. The data show that $N$. carpocapsae was widely distributed at that time mainly in lower areas of the Rhein-Main-District. Last instars $\left(\mathrm{L}_{5}\right)$ examined between 1972 and 1978 achieved high prevalences by $N$. carpocapsae in the first years in Frankfurt-Liederbach (62.0\%), Kriftel (57.5\%), Niederhofheim (up to 52.8\%) and Heidelberg-Kirchheim (74.0\%), with a certain decrease in subsequent years (Table 4). In diagnosed adults (Table 5), the infestation rates during 16 years in Frankfurt, for example, ranged between 48.2\% in 1975 and $33.6 \%$ in 1990 (minimum: 33.0\%/1983; maximum: 59.7\%/1978), during five years in Geisenheim, between 49.8\% in 1973 and 51.7\% in 1977 (minimum: 45.5\%/1976; maximum: 59.9\%/1974) and during 15 years in Kriftel between $30.4 \%$ in 1973 and 30.8\% in 1987 (minimum: 16.8\%/1982; maximum: $57.3 \% / 1985)$. Thus, generally, rather constant prevalences were noticed over the years. In Bayern, the number of infested adults caught in light traps was between $0 \%$ and $33.3 \%$, but altogether lower, as compared to Hessen. In the adults received from Baden-Württemberg, only one accession from Heidelberg showed a high prevalence of 50.6\% (1975).

Table 4. Occurrence and prevalence of the microsporidium, Nosema carpocapsae, in larvae of $C$. pomonella, from various regions in Germany (Hessen, Bayern and Baden-Württemberg; 1972-1978).

\begin{tabular}{|l|c|c|c|c|c|c|c|}
\hline Origin & \multicolumn{6}{|c|}{ Year-Infestation in \% and number of individuals (n) } \\
\hline Hessen (Rhein-Main area) & $\mathbf{1 9 7 2}$ & $\mathbf{1 9 7 3}$ & $\mathbf{1 9 7 4}$ & $\mathbf{1 9 7 5}$ & $\mathbf{1 9 7 6}$ & $\mathbf{1 9 7 7}$ & $\mathbf{1 9 7 8}$ \\
\hline Billings & & & $0(20)$ & & & & \\
\hline Darmstadt & $57.1(7)$ & & & & & & \\
\hline Frankfurt-Liederbach & & $62.0(50)$ & & & $41.5(94)$ & & \\
\hline Kriftel & & $57.5(80)$ & $37.0 *(309)$ & $30.8 *(100)$ & $17.9 *(140)$ & $36.8 *(36)$ & \\
\hline Niederhofheim & & $35.3(122)$ & $43.4 *(237)$ & $40.0(30)$ & $22.9(70)$ & & \\
\hline Bayern & & & & & & & \\
\hline Pittersberg & & & & & $29.8(94)$ & & \\
\hline Baden-Württemberg & & & & & & & \\
\hline Dossenheim & & & $26.3 *(103)$ & $18.1 *(791)$ & $17.3(110)$ & & \\
\hline Heidelberg-Kirchheim & & $74.0(49)$ & $50.3 *(79)$ & $33.3(30)$ & & & \\
\hline Karlsruhe-Augustenberg & & & & & & $3.0(30)$ & $20.0(35)$ \\
\hline Weinheim & & & $23.3(30)$ & & & & \\
\hline
\end{tabular}

* Means from several trappings. 
Table 5. Occurrence and prevalence of the microsporidium, Nosema carpocapsae, in adults of C. pomonella, from various regions in Germany (Hessen, Bayern and Baden-Württemberg) captured by light traps and pheromone traps, as well as from hibernation cages (1972-1990).

\begin{tabular}{|c|c|c|c|c|c|c|c|c|c|c|c|c|c|c|c|c|c|c|}
\hline \multirow{2}{*}{$\begin{array}{l}\quad \text { Origin } \\
\text { Hessen } \\
\text { (Rhein-Main } \\
\text { area) }\end{array}$} & \multicolumn{18}{|c|}{ Year-Infestation in \% and number of individuals (n) } \\
\hline & 1972 & 1973 & 1974 & 1975 & 1976 & 1977 & 1978 & 1979 & 1980 & 1981 & 1982 & 1983 & 1984 & 1985 & 1986 & 1987 & 1989 & 1990 \\
\hline $\begin{array}{l}\text { Darmstadt } \\
\text { (light trap) }\end{array}$ & $\begin{array}{r}23.9 \\
(117) \\
\end{array}$ & & & & & & $\begin{array}{l}27.9 \\
(147) \\
\end{array}$ & $\begin{array}{r}26.0 \\
(100)\end{array}$ & & & & & & & & & & \\
\hline $\begin{array}{l}\text { Frankfurt } \\
\text { (light trap) }\end{array}$ & & & & $\begin{array}{l}48.2 \\
(170) \\
\end{array}$ & $\begin{array}{r}55.5 \\
(321) \\
\end{array}$ & $\begin{array}{l}44.0 \\
(275) \\
\end{array}$ & $\begin{array}{l}59.7 \\
(139) \\
\end{array}$ & $\begin{array}{l}34.4 \\
(206) \\
\end{array}$ & $\begin{array}{l}44.4 \\
(187) \\
\end{array}$ & $\begin{array}{l}38.5 \\
(377) \\
\end{array}$ & $\begin{array}{r}46.6 \\
(253) \\
\end{array}$ & $\begin{array}{l}33.0 \\
(264) \\
\end{array}$ & $\begin{array}{l}42.4 \\
(184)\end{array}$ & $\begin{array}{r}46.8 \\
(171) \\
\end{array}$ & $\begin{array}{l}37.2 \\
(204) \\
\end{array}$ & $\begin{array}{l}34.4 \\
(90) \\
\end{array}$ & $\begin{array}{l}43.5 * \\
(361) \\
\end{array}$ & $\begin{array}{l}33.6 \\
(235) \\
\end{array}$ \\
\hline $\begin{array}{l}\text { Geisenheim } \\
\text { (light trap) }\end{array}$ & & $\begin{array}{l}49.8 * \\
(253)\end{array}$ & $\begin{array}{l}59.9 * \\
(372)\end{array}$ & $\begin{array}{l}55.4 \\
(186)\end{array}$ & $\begin{array}{l}45.5 \\
(110)\end{array}$ & $\begin{array}{l}51.7 \\
(29)\end{array}$ & & & & & & & & & & & & \\
\hline $\begin{array}{l}\text { Kriftel } \\
\text { (hibernation cages) }\end{array}$ & & $\begin{array}{l}50.0 \\
(12) \\
\end{array}$ & $\begin{array}{l}50.0 \\
(28) \\
\end{array}$ & $\begin{array}{l}45.0 \\
(20) \\
\end{array}$ & $\begin{array}{l}51.5 \\
(33) \\
\end{array}$ & & $\begin{array}{c}0 \\
(1) \\
\end{array}$ & & & & & & & & & & & \\
\hline $\begin{array}{l}\text { Kriftel } \\
\text { (light trap) }\end{array}$ & & $\begin{array}{l}30.4 * \\
(303) \\
\end{array}$ & $\begin{array}{l}31.7 \\
(202)\end{array}$ & $\begin{array}{l}45.4 \\
(77) \\
\end{array}$ & $\begin{array}{c}38.1 \\
(189) \\
\end{array}$ & $\begin{array}{l}22.4 \\
(112) \\
\end{array}$ & $\begin{array}{l}29.5 \\
(61) \\
\end{array}$ & $\begin{array}{l}31.7 \\
(63) \\
\end{array}$ & $\begin{array}{l}29.0 \\
(48) \\
\end{array}$ & $\begin{array}{l}22.3 \\
(94) \\
\end{array}$ & $\begin{array}{r}16.8 \\
(125) \\
\end{array}$ & $\begin{array}{l}18.7 \\
(112) \\
\end{array}$ & $\begin{array}{l}22.9 \\
(135)\end{array}$ & $\begin{array}{l}57.3 \\
(75) \\
\end{array}$ & $\begin{array}{l}25.8 \\
(124) \\
\end{array}$ & $\begin{array}{l}30.8 \\
(39) \\
\end{array}$ & & \\
\hline $\begin{array}{l}\text { Kriftel } \\
\text { (pheromone trap) }\end{array}$ & & & & $\begin{array}{l}30.7 \\
(319) \\
\end{array}$ & $\begin{array}{l}35.5 \\
(284)\end{array}$ & $\begin{array}{l}18.1 \\
(343)\end{array}$ & $\begin{array}{l}25.3 \\
(162) \\
\end{array}$ & $\begin{array}{l}25.5 \\
(153)\end{array}$ & $\begin{array}{l}23.7 \\
(156)\end{array}$ & & & & & & & & & \\
\hline $\begin{array}{l}\text { Langensel-bold } \\
\text { (pheromone trap) }\end{array}$ & & & & $\begin{array}{l}38.0 \\
(50) \\
\end{array}$ & & & & & & & & & & & & & & \\
\hline $\begin{array}{l}\text { Nordenstadt } \\
\text { (light trap) }\end{array}$ & & & & & & & & $\begin{array}{c}0 \\
(13) \\
\end{array}$ & & & & & & & & & & \\
\hline \multicolumn{19}{|l|}{ Bayern } \\
\hline $\begin{array}{l}\text { Deutenhofen } \\
\text { (light trap) }\end{array}$ & & $\begin{array}{l}20.5 * \\
(259)\end{array}$ & $\begin{array}{l}24.0 \\
(50) \\
\end{array}$ & $\begin{array}{l}23.5 \\
(51) \\
\end{array}$ & & & & & & & & & & & & & & \\
\hline $\begin{array}{l}\text { Erlabrunn } \\
\text { (light trap) } \\
\end{array}$ & & $\begin{array}{r}6.3 \\
(16) \\
\end{array}$ & $\begin{array}{l}20.0 \\
(15) \\
\end{array}$ & $\begin{array}{l}11.9 \\
(76) \\
\end{array}$ & $\begin{array}{r}11.7 \\
(170) \\
\end{array}$ & & & & & & & & & & & & & \\
\hline $\begin{array}{l}\text { Neuhaus } \\
\text { (light trap) }\end{array}$ & & $\begin{array}{c}0 \\
(18)\end{array}$ & $\begin{array}{c}0 \\
(11)\end{array}$ & $\begin{array}{c}0 \\
(15)\end{array}$ & $\begin{array}{c}33.0 \\
(3)\end{array}$ & & & & & & & & & & & & & \\
\hline
\end{tabular}


Table 5. Cont.

\begin{tabular}{|c|c|c|c|c|c|c|c|c|c|c|c|c|c|c|c|c|c|c|}
\hline Origin & & & & & & Yea & Infesta & tion in & $\%$ and & lumbe & of ind & iduals ( & & & & & & \\
\hline $\begin{array}{l}\text { Hessen } \\
\text { (Rhein-Main area) }\end{array}$ & 1972 & 1973 & 1974 & 1975 & 1976 & 1977 & 1978 & 1979 & 1980 & 1981 & 1982 & 1983 & 1984 & 1985 & 1986 & 1987 & 1989 & 1990 \\
\hline $\begin{array}{l}\text { Igensdorf } \\
\text { (light trap) }\end{array}$ & & $\begin{array}{c}0 \\
(3) \\
\end{array}$ & & & & & & & & & & & & & & & & \\
\hline $\begin{array}{l}\text { Eichelsdorf } \\
\text { (light trap) }\end{array}$ & & $\begin{array}{c}0 \\
(35)\end{array}$ & $\begin{array}{l}10.0 \\
(10)\end{array}$ & & & & & & & & & & & & & & & \\
\hline $\begin{array}{l}\text { Mallersdorf } \\
\text { (light trap) }\end{array}$ & & & $\begin{array}{l}27.3 \\
(11)\end{array}$ & $\begin{array}{c}33.3 \\
(3)\end{array}$ & & & & & & & & & & & & & & \\
\hline $\begin{array}{l}\text { Uffenheim } \\
\text { (light trap) }\end{array}$ & & & $\begin{array}{c}33.3 \\
(3)\end{array}$ & $\begin{array}{l}14.3 \\
(21)\end{array}$ & $\begin{array}{l}19.0 \\
(21)\end{array}$ & & & & & & & & & & & & & \\
\hline $\begin{array}{l}\text { Weihen-stephan } \\
\text { (light trap) }\end{array}$ & & & & & $\begin{array}{l}100 \\
(5)\end{array}$ & & & & & & & & & & & & & \\
\hline Baden-Württembe & & & & & & & & & & & & & & & & & & \\
\hline $\begin{array}{l}\text { Dossenheim } \\
\text { (light trap) }\end{array}$ & & & $\begin{array}{r}28.4 * \\
(123)\end{array}$ & $\begin{array}{r}24.1 * \\
(112)\end{array}$ & & & & & & & & & & & & & & \\
\hline $\begin{array}{l}\text { Dossenheim } \\
\text { (hibernation cages) }\end{array}$ & & & & & & & & & & & & $\begin{array}{l}20.8 * \\
(120)\end{array}$ & & & & & & \\
\hline $\begin{array}{l}\text { Heidelberg } \\
\text { (pheromone trap) }\end{array}$ & & & & $\begin{array}{l}50.6 \\
(16)\end{array}$ & & & & & & & & & & & & & & \\
\hline $\begin{array}{l}\text { Ludwigsburg } \\
\text { (pheromone trap) }\end{array}$ & & & & $\begin{array}{c}0 \\
(27)\end{array}$ & & & & & & & & & & & & & & \\
\hline
\end{tabular}

* Means from several trappings. 
Experiments on the effect of a microsporidian infection on the fecundity and fertility of C. pomonella revealed significant differences between healthy and infested female adults (Figure 2). The mean egg number of healthy females was 105 per individual, compared to only 64 of females infected by $N$. carpocapsae. Similarly, the hatching rate of egg samples of healthy females was $64 \%$ compared to only $46 \%$ of those laid by infected females (see, also, [25]).

Figure 2. Comparison of fecundity and fertility data from healthy and microsporidia-infected codling moth adults. Box plot with median (solid line) and arithmetic mean (- - -) and the 5 and $95 \%$ percentile $(+)$, respectively. Means of the eggs and neonate larvae and the angular transformed values of the hatching rate with diverse letters are significantly different (Student-Newman-Keuls test, $p \leq 0.05$ ).

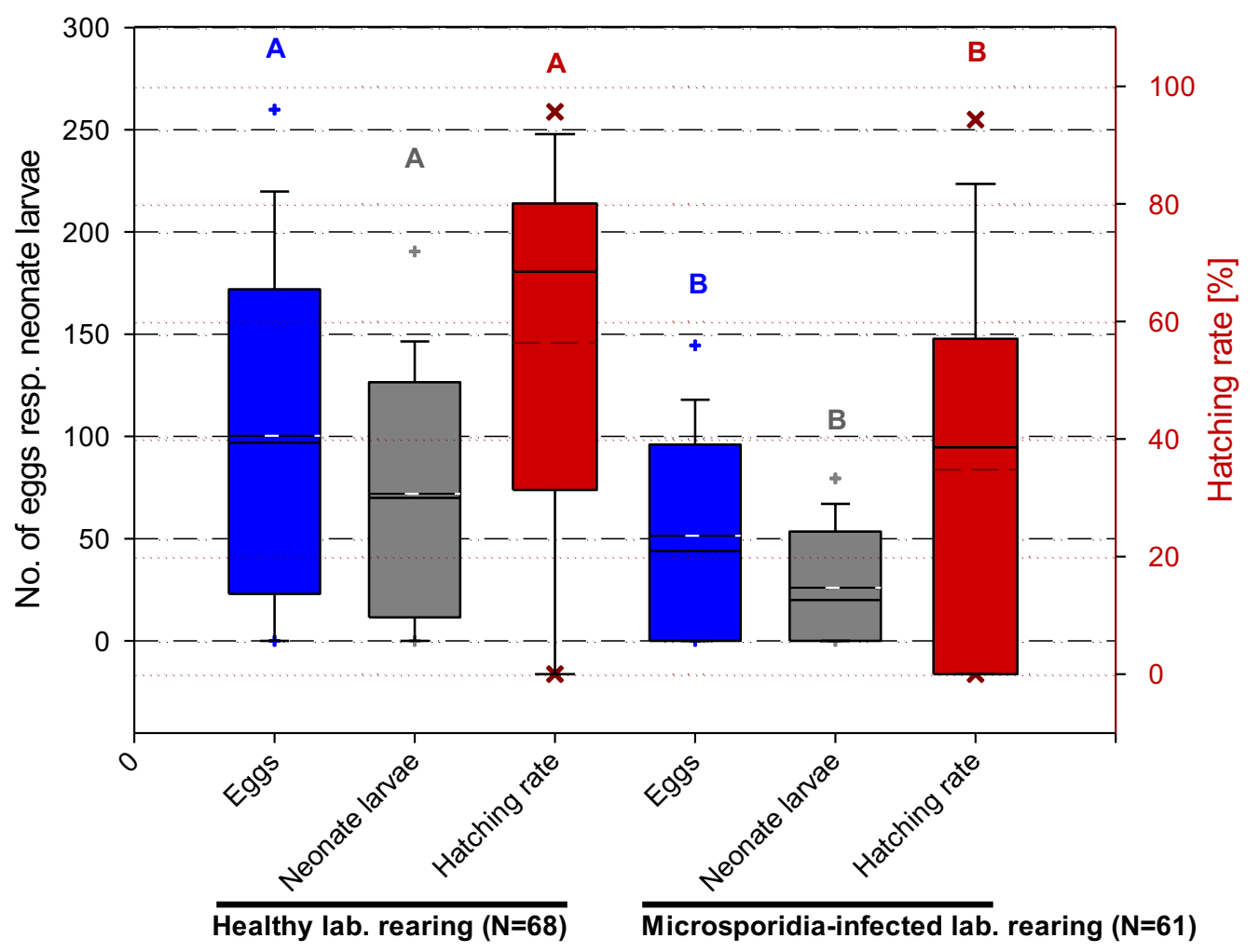

\section{Discussion}

The diagnostic studies represent an overview on entomopathogenic and other microorganisms, including nematodes, found in larvae, pupae and adults of the codling moth over a longer period of time. Except for $N$. carpocapsae, the investigations were not conducted systematically by routine examination of field populations, but irregularly whenever codling moth specimens were sent to the Institute for Biological Control for diagnosis of their diseases.

The most frequently diagnosed entomopathogens were the codling moth granulovirus ( $\mathrm{CpGV})$, various fungi, especially B. bassiana and I. farinosa, and N. carpocapsae. Long-term prognostic studies on the prevalence of $N$. carpocapsae in larvae and adults in various areas of Germany document the state and development of infestation in different field populations.

$\mathrm{CpGV}$ was only found in larvae and pupae from laboratory colonies and in field populations where the virus was applied for plant protection. The reason for the occurrence of $\mathrm{CpGV}$ in laboratory reared 
larvae is not always clear. Probably, they are contaminated accidentally by handling. Thus, a natural occurrence of $\mathrm{CpGV}$, so far, is not proven in Germany, Austria or Switzerland. Certainly, it is difficult to find infected larvae in the field, as they rapidly decay. Whether a propagation of CpGV from virus-treated orchards to other untreated populations occurs, e.g., by contaminated flying insects, by birds or by atmospheric conditions, is scarcely investigated [62-64]. The first detection of CpGV was in 1963 in an orchard in Mexico [65].

The role of the observed unidentified bacteria, spore-formers or Serratia spp. is unclear. They may be part of the normal gut flora, but some of them also may be pathogens. During epizootics in different laboratory colonies of $C$. pomonella in Poland, several strains of the well-known pathogen, B. thuringiensis, were isolated [20]. In the present study, however, B. thuringiensis was not found. In any case, further investigations on the effect of these microorganisms on individuals and on field populations of $C$. pomonella are necessary.

Entomopathogenic fungi and, especially, B. bassiana and I. farinosa, were the major and most frequent group of pathogens diagnosed in last instars and diapaused larvae and pupae. The frequent occurrence and the high prevalence of $B$. bassiana show that this species is an important naturally occurring mortality factor, mainly in hibernating populations, which obviously is permanently present in orchard ecosystems. This fungus has often been found in C. pomonella and other tortricids [7,18,30-32,34,66]. In an orchard in Austria (Steiermark), Russ [31] observed an outbreak of $B$. bassiana in diapausing larvae with prevalences of $77 \%$, while in other more dry locations, only $1.3 \%$ and $2.5 \%$ of the larvae were infected. During investigations on diapausing larvae and in pupae of the codling moth in the south of Sweden, besides different parasitoids, mainly B. bassiana (34.4\%) and I. farinosa (29.5\%) were detected [34]. In Nova Scotia, Canada, however, Jacques and MacLellan [66] found that fungi only killed 1.7\%, with a maximum of $10 \%$ of diapausing larvae of $C$. pomonella populations. According to our knowledge, no detailed investigations on the role of entomopathogenic fungi as natural mortality factors and their impact on field populations in Germany and other European countries are available. Furthermore, it is unknown where and when larvae of the codling moth become infected by B. bassiana or I. farinosa in apple trees, i.e., also studies on the ecology of these two fungi in the field are necessary to clarify their role as natural mortality factors. In addition, investigations on these fungi as pathogens of codling moth larvae from conventionally and ecologically treated apple orchards of different regions would be important to determine possible impacts of other plant protection treatments (e.g., fungicides or herbicides) on the prevalence of these antagonists.

The most frequently identified pathogen of codling moth larvae and adults was $N$. carpocapsae, which causes a chronic disease. For the first time, data are presented on the occurrence and prevalence of $N$. carpocapsae in C. pomonella populations from various locations of Germany over a long period of time. It was demonstrated that this microsporidium causes prevalences of living specimens up to $c a$. $70 \%$. The average larval infestation levels in various codling moth populations from different areas in Germany (Hessen, Bayern, Baden-Württemberg) ranged from about 20\% to 50\% (see, also, [25,33]), which documents a relatively prevalent occurrence at that time. Similar infection levels were ascertained in adults caught in light traps in Hessen over 18 years. This microsporidium was already observed in Germany in 1955 and, then, as documented here, in 1972 and in the following years in larvae, pupae and, particularly, also, in adults of $C$. pomonella (see, also, [33,57]). This is remarkable, 
as $N$. carpocapsae was reported for the first time in North America only in 2001 [8]. Huger [25,33] demonstrated that the chronic infection is transmitted transovarially from infected females to the progeny. There was no significant difference in the infestation rate of female and male adults by $N$. carpocapsae [67].

Significant differences in the fecundity and fertility of healthy and infected adults of C. pomonella were observed (Figure 1; [25]). Thus, in the infected batch, an average decrease of the number of hatched egg larvae by $56 \%$ was determined. Therefore, it can be concluded that, depending on the natural prevalence, $N$. carpocapsae also may have an appreciable impact in reducing field populations. However, data on the actual infestation rates of codling moth populations by this microsporidium are not available. Similar observations have been published by Andreadis [68] and Lewis et al. [69] for populations of the European corn borer, Ostrinia nubilalis, infected by Nosema pyrausta, resulting in a reduced fertility between $30 \%$ and $50 \%$.

Reduced fertility of adults caused by $N$. carpocapsae may also lead to some problems in laboratory reared colonies [25,70]. In contrast, Siegel et al. [8] did not find any effect of a North American isolate of $N$. carpocapsae on the fecundity of females; however, a higher mortality and longer developmental period compared to healthy larvae and pupae were noticed. Furthermore, they showed that the first hatched larvae from one egg mass nearly were uninfected. Negative effects of microsporidia on the rate of progeny were also documented for the European corn borer, O. nubilalis infected by N. pyrausta [71] and the gypsy moth, Lymantria dispar, infected by Vairimorpha disparis and Nosema lymantriae [72-76], where an increase in the mortality of larvae was noted. No effect of $N$. carpocapsae on the mortality of diapausing larvae of C. pomonella was observed [33], while there was a clear correlation between the mortality of diapausing larvae of $O$. nubilalis and their infestation by N. pyrausta [77].

In addition to these direct effects of $N$. carpocapsae on its host, $C$. pomonella, various interactions between this microsporidium and other antagonists are known. Experiments on the co-occurrence of $N$. carpocapsae and $\mathrm{CpGV}$ in laboratory colonies revealed that microsporidia-infected individuals are six-times less susceptible to the CpGV compared to healthy ones [78]. The effects of such double infections to codling moth populations under field conditions are unknown. Generally, microsporidia are rather selective entomopathogens with a small host range. However, in a laboratory colony of the codling moth parasitoid, Ascogaster quadridentata (Hymenoptera, Braconidae), it was observed that $N$. carpocapsae not only infects $C$. pomonella, but also the egg-parasitoid itself, thus resulting in a collapse of the rearing [79,80]. Furthermore, an infection of the egg-parasitoid, Trichogramma evanescens, by $N$. carpocapsae was proven, causing a reduction of the parasitic capacity of the microsporidia-infected Trichogramma females [81]. Possible interactions of $N$. carpocapsae with these parasitoids and their effect on codling moth populations in the field and their ecological relevance are not yet investigated.

Diagnostic studies on the occurrence and prevalence of naturally occurring insect pathogens of C. pomonella are important, also with regard to their possible use as biocontrol agents. For example, a strain of M. anisopliae (M.a. 43, JKI fungus collection) isolated from a codling moth larva in 1971 originating from Austria was successfully used under the strain names, F52 or BIPESCO 5, against other pest insects, e.g., the black vine weevil, Otiorhynchus sulcatus (Coleoptera: Curculionidae), and 
was the active ingredient of the former mycoinsecticide, "BIO 1020", of the company, BAYER [82] or against white grubs of the garden chafer [83].

So far, only the CpGV is commercialized for codling moth control (e.g., [3]), but recently, also, entomoparasitic nematodes (Steinernema carpocapsae and S. feltiae) were tested successfully $[7,15,27,29,84,85]$. Formerly, also, the fungus, B. bassiana, was tested in laboratory and field experiments $[7,21,23,24,26]$. As already suggested [21] and according to our findings of the fungus in the last larval or diapausing instars, an application on branches and stems in late summer or autumn seems to be most promising, as well as a soil application under trees to infect overwintering larvae and to increase the antagonistic potential. In contrast to these insect pathogens, $N$. carpocapsae was never used as a biocontrol agent against $C$. pomonella. However, other microsporidia, such as Nosema pyrausta, were successfully introduced in field populations of the European corn borer, Ostrinia nubilalis [68,86-88]. Furthermore, the microsporidia, Nosema sp. and Vavraia sp., could be established in populations of the gypsy moth, Lymantria dispar [89].

\section{Conclusions}

The results of these long-term diagnostic and other investigations show that C. pomonella is attacked by several entomopathogens that are important mortality factors. However, the knowledge on the impact of these microbial antagonists and on possible interactions with other organisms in field populations is scarce. Therefore, the data presented here may serve as a useful base for further systematic studies on the ecology, natural occurrence and prevalence of these entomopathogens in codling moth populations. With regard to increasing resistance problems and the aimed reduction of chemical pesticides, an assessment of their potential and of the possibilities for their conservation and augmentation in the field is important with respect to the development of new strategies for biological or integrated control of $C$. pomonella.

\section{Acknowledgments}

We thank all former colleagues, technicians and senders of codling moth specimens, especially Zotzmann (formerly Plant Protection Office Frankfurt/Main), for their collaboration. We are also grateful to Stephan, Julius Kühn Institute, Darmstadt, for statistical analyses.

\section{Conflict of Interest}

The authors declare no conflict of interest.

\section{References}

1. Alford, D.V. Farbatlas der Obstschädlinge: Erkennung, Lebensweise und Bekämpfung; Publisher F. Enke: Stuttgart, Germany, 1987; pp. 170-171.

2. Galli, P.; Epp, P. Pheromonfallen zur Flugüberwachung des Apfelwicklers. Obstbau 2006, 31, 280-282.

3. Kienzle, J.; Gernoth, H.; Litterst, M.; Huber, J.; Zebitz, C.P.W.; Jehle, J.A. Biologie und Wirkungsweise des Apfelwickler-Granulovirus. Obstbau 2006, 31, 284-286. 
4. Litterst, M.; Gernoth, H.; Fried, A. Pflanzenschutzmittel zur Bekämpfung des Apfelwicklers. Obstbau 2006, 31, 282-283.

5. Trautmann, M.; Scheer, C. Einsatz der Verwirrungsmethode zur biotechnischen Bekämpfung des Apfelwicklers am Bodensee (1996-2005). Obstbau 2006, 31, 287-290.

6. Witzgall, P.; Stelinski, L.; Gut, L.; Thomson, D. Codling moth management and chemical ecology. Ann. Rev. Entomol. 2008, 53, 503-522.

7. Cross, J.V.; Solomon, M.G.; Chandler, D.; Jarrett, P.; Richardson, P.N.; Winstanley, D.; Bathon, H.; Huber, J.; Keller, B.; Langenbruch, G.-A.; et al. Biocontrol of pests of apples and pears in Northern and Central Europe: 1. Microbial agents and nematodes. Biocontrol Sci. Technol. 1999, 9, 125-149.

8. Siegel, J.P.; Lacey, L.A.; Vossbrinck, C.R. Impact of a North American isolate of the microsporidium Nosema carpocapsae on a laboratory population of the codling moth, Cydia pomonella. J. Invertebr. Pathol. 2001, 78, 244-250.

9. Charmillot, P.J.; Pasquier, D. Progression de la résistance du carpocapse Cydia pomonella aux insecticides. Rev. Suisse Vitic. Arboric. Hortic. 2002, 34, 95-100.

10. Charmillot, P.J.; Pasquier, D. Combination of mating disruption (MD) technique and granulosis virus to control resistant strains of codling moth Cydia pomonella. Bull. OILB/SROP 2003, 26, $27-29$.

11. Schmitt, A.; Bisutti, I.L.; Ladurner, E.; Benuzzi, M.; Sauphanor, B.; Kienzle, J.; Zingg, D.; Undorf-Spahn, K.; Fritsch, E.; Huber, J.; et al. The occurrence and distribution of resistance of codling moth to Cydia pomonella granulovirus in Europe. J. Appl. Entomol. 2013, doi:10.1111/jen.12046.

12. Lacey, L.A.; Unruh, T.R. Biological control of codling moth (Cydia pomonella, Lepidoptera: Tortricidae) and its role in integrated pest management, with emphasis on entomopathogens. Vedalia 2005, 12, 33-60.

13. Charmillot, P.J.; Pasquier, D.; Salamin, C.; Briand, F.; Ter-Hovannesyan, A.; Azizian, A.; Kutinkova, H.; Peeva, P.; Velcheva, N. Détection de la résistance du carpocapse Cydia pomonella. Tests d'insecticides sur les chénilles diapausantes de Suisse, d'Armenie et de Bulgarie. Rev. Suisse Vitic. Arboric. Hortic. 2007, 39, 385-389.

14. Asser-Kaiser, S.; Fritsch, E.; Undorf-Spahn, K.; Kienzle, J.; Eberle, K.E.; Gund, N.A.; Reineke, A.; Zebitz, C.P.W.; Heckel, D.G.; Huber, J.; et al. Rapid emergence of baculovirus resistance in codling moth due to dominant sex-linked inheritance. Science 2007, 317, 1916-1918.

15. Lacey, L.A.; Shapiro-Ilan, D.I. Microbial control of insect pests in temperate orchard systems: Potential for incorporation into IPM. Ann. Rev. Entomol. 2008, 53, 121-144.

16. Undorf-Spahn, K.; Fritsch, E.; Huber, J.; Kienzle, J.; Zebitz, C.P.; Jehle, J.A. High stability and no fitness costs of the resistance of codling moth to Cydia pomonella granulovirus (CpGV-M). J. Invertebr. Pathol. 2012, 111, 136-142.

17. Falcon, L.A.; Huber, J. Biological control of the codling moth. In Tortricid Pests, Their Biology, Natural Enemies and Control; van der Geest, L.P.S., Evenhuis, H.H., Eds.; Elsevier Science Publishers: Amsterdam, The Netherlands, 1991; pp. 355-369. 
18. Zimmermann, G.; Weiser, J. Pathogens and diseases. In Tortricid Pests, Their Biology, Natural Enemies and Control; van der Geest, L.P.S., Evenhuis, H.H., Eds.; Elsevier Science Publishers: Amsterdam, The Netherlands, 1991; Chapter 3.2, pp. 253-271.

19. Lacey, L.A.; Shapiro-Ilan, D.I. The potential role for microbial control of orchard insect pests in sustainable agriculture. J. Food Agric. Environ. 2003, 1, 326-331.

20. Konecka, E.; Kaznowski, A.; Ziemnicka, J.; Kiemnicki, K. Molecular and phenotypic characterisation of Bacillus thuringiensis isolated during epizootics in Cydia pomonella L. J. Invertebr. Pathol. 2007, 94, 56-63.

21. Müller-Kögler, E. Pilzkrankheiten bei Insekten. Anwendung zur Biologischen Schädlingsbekämpfung und Grundlagen der Insektenmykologie; Publisher P. Parey: Berlin, Germany, 1965.

22. Burges, H.G., Hussey, N.W., Eds.; Microbial Control of Insects and Mites; Academic Press: London, UK, 1971.

23. Ferron, P. Pest Control by the Fungi Beauveria and Metarhizium. In Microbial Control of Pests and Plant Diseases 1970-1980; Burges, H.D., Ed.; Academic Press: London, UK, 1971; pp. 465-482.

24. Ferron, P.; Vincent, J.J. Preliminary experiments on the use of Beauveria bassiana against Carpocapsa pomonella. Mitt. Biologischen Bundesanstalt Land Forstwirtschaft Berlin Dahlem 1978, 180, 84-87.

25. Huger, A.M. Influence of a Microsporidian Disease on Fecundity and Fertility on the Codling Moth; Annual Report of the Federal Biological Research Centre for Agriculture and Forestry: Braunschweig, Germany, 1978; pp. H90-H91.

26. Audemard, H.; Ferron, P. Codling moth control with Beauveria bassiana in orchards. IOBC/WPRS Bull. 1980, 3, 55-56.

27. Peters, A.; Katz, P.; Elias, E. Entomopathogenic nematodes for biological control of codling moth. In Proceedings of the 13th International Conference on Cultivation Technique and Phytopathological Problems in Organic Fruit-Growing, Weinsberg, Germany, 18-20 February 2008; pp. 284-286.

28. Kienzle, J.; Heinisch, D.; Kiefer, J.; Trautmann, M.; Volk, F.; Zimmer, J.; Zebitz, C.P.W. Three years experience with entomopathogenic nematodes for the control of overwintering codling moth larvae in different regions of Germany. In Proceedings of the 14th International Conference on Organic Fruit-Growing, Hohenheim, Germany, 22-24 February 2010; pp. 163-168.

29. Lacey, L.A.; Shapiro-Ilan, D.I.; Glenn, G.M. Post-application of anti-desiccant agents improves efficacy of entomopathogenic nematodes in formulated host cadavers or aqueous suspension against diapausing codling moth larvae (Lepidoptera: Tortricidae). Biocontrol Sci. Technol. 2010, 20, 909-921.

30. Michelbacher, A.E.; Middlekauff, W.W.; Hansen, C. Occurrence of a fungus disease in overwintering stages of the codling moth. J. Econ. Entomol. 1950, 43, 955-956.

31. Russ, K. Über ein bemerkenswertes Auftreten von Beauveria bassiana (Bals.) Vuill. an Carpocapsa pomonella (L.). Pflanzenschutzberichte 1964, 31, 105-108.

32. Müller-Kögler, E. Insektenpathogene Pilze von Apfelwicklerraupen; Annual Report of the Federal Biological Research Centre for Agriculture and Forestry: Braunschweig, Germany, 1971; p. P79. 
33. Huger, A.M. Diagnostische Untersuchungen über das Auftreten von Krankheiten in Freilandpopulationen Wichtiger Schadinsekten; Annual Report of the Federal Biological Research Centre for Agriculture and Forestry: Braunschweig, Germany, 1976; pp. H79-H80.

34. Subinprasert, S. Natural enemies and their impact on overwintering codling moth populations (Laspeyresia pomonella L.) (Lep., Tortricidae) in South Sweden. J. Appl. Entomol. 1987, 103, $46-55$.

35. Glen, D.M.; Milsom, N.F. Survival of mature larvae of codling moth (Cydia pomonella) on apple trees and ground. Ann. Appl. Biol. 1978, 90, 133-146.

36. Zelger, R.; Harzer, U.; Epp, P.; Trautmann, M. Untersuchungen zur Überwinterung des Apfelwicklers. Obstbau 2006, 31, 262-264.

37. Kleespies, R.G.; Huger, A.M.; Zimmermann, G. Diseases of insects and other arthropods: Results of diagnostic research over 55 years. Biocontrol. Sci. Technol. 2008, 18, 439-484.

38. Kleespies, R.G.; Huger, A.M.; Zimmermann, G. Database on Arthropod Diseases. Available online: http://arthropodenkrankheiten.jki.bund.de/ (accessed on 18 March 2009).

39. Huger, A.M. Histologie und Diagnose als praxisbezogene Grundlagenforschung im biologischen Pflanzenschutz. Gesunde Pflanzen 1970, 22, 36-40.

40. Huger, A.M. Methoden und Bedeutung der Diagnosen von Insektenkrankheiten. Z. Pflanzenkr. Pflanzenschutz 1974, 81, 372-388.

41. Poinar, G.O., Jr.; Thomas, G.M. Diagnostic Manual for the Identification of Insect Pathogens; Plenum Press: New York, NY, USA, London, UK, 1978.

42. Burges, H.D., Ed.; Microbial Control of Pests and Plant Diseases 1970-1980; Academic Press: London, UK, 1981.

43. Plattner, H.; Zingsheim, H.-P. Elektronenmikroskopische Methodik in der Zell- und Molekularbiologie; Publisher G. Fischer: Stuttgart, Germany, 1987.

44. Romeis, B. Mikroskopische Technik; Publisher Urban \& Schwarzenberg: München, Germany, 1989.

45. Tanada, Y.; Kaya, H.K. Insect Pathology; Academic Press, Inc.: San Diego, CA, USA, 1993.

46. Lacey, L.A., Ed.; Manual of Techniques in Insect Pathology; Academic Press: San Diego, CA, USA, 1997.

47. Lacey, L.A., Ed.; Field Manual of Techniques in Invertebrate Pathology; Publisher Springer: Berlin, Heidelberg, Germany, 2000.

48. Kleespies, R.G.; Vossbrinck, C.R.; Lange, M.; Jehle, J.A. Morphological and molecular investigations of a microsporidium infecting the European grape vine moth, Lobesia botrana Den. et Schiff., and its taxonomic determination as Cystosporogenes legeri nov. comb. J. Invertebr. Pathol. 2003, 83, 240-248.

49. Lacey, L.A., Kaya, H.K., Eds.; Field Manual of Techniques in Insect Pathology; 2nd ed.; Publisher Springer: Berlin, Heidelberg, Germany, 2007.

50. Kleespies, R.G.; Marshall, S.D.G.; Schuster, C.; Townsend, R.J.; Jackson, T.; Leclerque, A. Genetic and electron-microscopic characterization of Rickettsiella bacteria from the manuka beetle, Pyronota setosa (Coleoptera: Scarabaeidae). J. Invertebr. Pathol. 2011, 107, 206-211.

51. Barnett, H.L. Illustrated Genera of Imperfect Fungi, 2nd ed.; Burgess Publishing Company: Minneapolis, MN, USA, 1960. 
52. Barron, G.L. The Genera of Hyphomycetes from Soil; The Williams \& Wilkins Company: Baltimore, MD, USA, 1968.

53. Humber, R.A. Fungi: Identification. In Manual of Techniques in Insect Pathology; Lacey, L.A., Ed.; Academic Press: San Diego, CA, USA, 1997; pp. 153-185.

54. Humber, R.A. Identification of entomopathogenic fungi. In Manual of Techniques in Invertebrate Pathology, 2nd ed.; Lacey, L.A., Ed.; Academic Press: London, UK, 2012; pp. 151-187.

55. Luangsa-Ard, J.J.; Hywel-Jones, N.L.; Manoch, L.; Samson, R.A. On the relationships of Paecilomyces sect. Isarioidea species. Mycol. Res. 2005, 109, 581-589.

56. Sung, G.H.; Hywel-Jones, N.L.; Sung, J.M.; Luangsa-Ard, J.J.; Shrestha, B.; Spatafora, J.W. Phylogenetic classification of Cordyceps and the clavicipitaceous fungi. Stud. Mycol. 2007, 57, 5-59.

57. Huger, A.M. Erhebungen über infektiöse Erkrankungen in Freilandpopulationen des Apfelwicklers; Annual Report of the Federal Biological Research Centre for Agriculture and Forestry: Braunschweig, Germany, 1973; p. 90.

58. Huger, A.M. Federal Biological Research Centre for Agriculture and Forestry, Darmstadt, Germany. Unpublished work, 1972-1990.

59. Weiss, L.M.; Edlind, T.D.; Vossbrinck, C.R.; Hashimoto, T. Microsporidian molecular phylogeny: The fungal connection. J. Eukaryot. Microbiol. 1999, 46, 17S-18S.

60. Corradi, N.; Slamovits, C.H. The intriguing nature of microsporidian genomes. Brief. Funct. Genomics 2011, 10, 115-124.

61. Posada, F.; Vega, F.E.; Rehner, S.A.; Blackwell, M.; Weber, D.; Suh, S.O.; Humber, R.A. Syspastospora parasitica, a mycoparasite of the fungus Beauveria bassiana attacking the Colorado potato beetle Leptinotarsa decemlineata: A tritrophic association. J. Insect Sci. 2004, 4, 24.

62. Szalay-Marzso, L.; Vago, C. Transmission of baculovirus by mites. Study of granulosis virus of codling moth (Laspeyresia pomonella L.). Acta Phytopathol. Acad. Sci. Hung. 1975, 10, 113-122.

63. Sheppard, R.F.; Stairs, G.R. Effects of dissemination of low dosage levels of a granulosis virus in populations of the codling moth. J. Econ. Entomol. 1976, 69, 583-586.

64. Pultar, O.; Kocourek, F.; Berankova, J.; Stara, J.; Kuldova, J.; Hrdy, I. Codling moth management by means of pheromone stations with Cydia pomonella granulosis virus. In Proceedings of the International Conference on Integrated Fruit Production, Leuven, Belgium, 27 July-1 August 1998; pp. 477-480.

65. Tanada, Y. A granulosis virus of the codling moth, Carpocapsa pomonella (Linnaeus) (Olethreutidae, Lepidoptera). J. Insect Pathol. 1964, 6, 378-380.

66. Jaques, R.P.; MacLellan, C.R. Fungal mortality of overwintering larvae of the codling moth in apple orchards in Nova Scotia. J. Invertebr. Pathol. 1965, 7, 291-296.

67. Huger, A.M. Federal Biological Research Centre for Agriculture and Forestry, Darmstadt, Germany. Unpublished work, 1973-1974.

68. Andreadis, T.G. Epizootiology of Nosema pyrausta in field populations of the European corn borer (Lepidoptera, Pyralidae). Environ. Entomol. 1984, 13, 882-887.

69. Lewis, L.C.; Sumerford, D.V.; Bing, L.A.; Gunnarson, R.D. Dynamics of Nosema pyrausta in natural populations of the European corn borer, Ostrinia nubilalis: A six-year study. BioControl 2006, 51, 627-642. 
70. Bathon, H. Zur Chemotherapie einer Mikrosporidiose an Apfelwicklern; Annual Report of the Federal Biological Research Centre for Agriculture and Forestry: Braunschweig, Germany, 1974; pp. H88-H89.

71. Huger, A.M. Effects of a persistent microsporidiosis on the reproduction rate of the European corn borer (in German); Annual Report of the Federal Biological Research Centre for Agriculture and Forestry: Berlin, Braunschweig, Germany, 1980; pp. H84-H85.

72. Linde, A.; Richardt, K.; Bartsch, D.; Seidel, C. Evaluation of the potential of microsporidia for the regulation of gypsy moth populations (Lymantria dispar L.). Mitt. Dtsch. Ges. Allg. Ang. Entomol. 2000, 12, 127-131.

73. Goertz, D.; Pilarska, D.; Kereselidze, M.; Solter, L.F.; Linde, A. Studies on the impact of two Nosema isolates from Bulgaria on the gypsy moth (Lymantria dispar L.). J. Invertebr. Pathol. 2004, 87, 105-113.

74. Goertz, D.; Solter, L.F.; Linde, A. Horizontal and vertical transmission of a Nosema sp. (Microsporidia) from Lymantria dispar (L.) (Lepidoptera: Lymantriidae). J. Invertebr. Pathol. 2007, 95, 9-16.

75. Goertz, D.; Golldack, J.; Linde, A. Two different and sublethal isolates of Nosema lymantriae (Microsporidia) reduce the reproductive success of their host, Lymantria dispar. Biocontrol Sci. Technol. 2008, 18, 419-430.

76. Goertz, D.; Hoch, G. Horizontal transmission pathways of terrestrial microsporidia: A quantitative comparison of three pathogens infecting different organs in Lymantria dispar L. (Lep.: Lymantriidae) larvae. Biol. Control 2008, 44, 196-206.

77. Huger, A.M. Effects of a Persistent Microsporidiosis on the Survival Rate of Overwintering Populations of the European Corn Borer (in German); Annual Report of the Federal Biological Research Centre for Agriculture and Forestry: Berlin, Braunschweig, Germany, 1980; pp. H83-H84.

78. Huber, J. Wechselwirkung Zwischen der Granulose und einer Mikrosporidiose des Apfelwicklers; Annual Report of the Federal Biological Research Centre for Agriculture and Forestry: Berlin, Braunschweig, Germany, 1975; p. H82.

79. Huger, A.M.; Neuffer, G. Das Mißlingen einer Apfelwickler-Parasitenzucht; Annual Report of the Federal Biological Research Centre for Agriculture and Forestry: Berlin, Braunschweig, Germany, 1977; pp. H87-H88.

80. Huger, A.M.; Neuffer, G. Infection of the braconid parasite Ascogaster quadridentata (Hymenoptera: Braconidae) by a microsporidian of its host, Laspeyresia pomonella (in German). Mitt. Biologischen Bundesanstalt Land Forstwirtschaft 1978, 180, 105-106.

81. Huger, A.M. Investigations on the Effect of Microsporidium Nosema carpocapsae on Parasitizing Capacity of the Egg Parasitoid Trichogramma evanescens (in German); Annual Report of the Federal Biological Research Centre for Agriculture and Forestry: Berlin, Braunschweig, Germany, 1983; p. H81.

82. Reinecke, P.; Andersch, W.; Stenzel, K.; Hartwig, J. Bio-1020 A new microbial insecticide for use in horticultural crops. In Brighton Crop Protection Conference: Pests and Diseases; British Crop Protection Council: Farnham, England, UK, 1990; Volume 1, pp. 49-54. 
83. Pernfuss, B.; Zelger, R.; Kron-Morelli, R.; Strasser, H. Control of the garden chafer Phyllopertha horticola with GranMet-P, a new product made of Metarhizium anisopliae. IOBC/WPRS Bull. 2005, 28, 47-50.

84. Unruh, T.R.; Lacey, L.A. Control of codling moth, Cydia pomonella (Lepidoptera: Tortricidae), with Steinernema carpocapsae: Effects of sublemental wetting in pupation site on infection rate. Biol. Control 2001, 20, 48-56.

85. Curto, G.; Reggiani, A.; Vergnani, S.; Caruso, S.; Ladurner, E. Effectiveness of entomopathogenic nematodes in the control of Cydia pomonella larvae in Northern Italy. In Proceedings of the 13th International Conference on Cultivation Technique and Phytopathological Problems in Organic Fruit-Growing, Weinsberg, Germany, 18-20 February 2008; pp. 271-276.

86. Huger, A.M. Versuche zur Künstlichen Infektion von Gesunden Freilandpopulationen Wichtiger Schadinsekten, vor Allem mit Mikrosporidien; Annual Report of the Federal Biological Research Centre for Agriculture and Forestry: Berlin, Braunschweig, Germany, 1979; p. H84.

87. Kurtti, T.J.; Munderloh, U.G. Issues in the use of microsporidia for biological control of European corn borer. In Ecological Interactions and Biological Control; Andow, D.A., Ragsdale, D.W., Nyvall, R.F., Eds.; Westview Press: Boulder, CO, USA, 1997; pp. 195-214.

88. Lewis, L.C.; Bruck, D.J.; Prasifka, J.R.; Raun, E.S. Nosema pyrausta: Its biology, history, and potential role in a landscape of transgenic insecticidal crops. Biol. Control 2009, 48, 223-231.

89. Jeffords, M.R.; Maddox, J.V.; McManus, M.L.; Webb, R.E.; Wieber, A. Egg contamination as a method for the inoculative release of exotic microsporidia of the gypsy moth. J. Invertebr. Pathol. 1988, 51, 190-196.

(C) 2013 by the authors; licensee MDPI, Basel, Switzerland. This article is an open access article distributed under the terms and conditions of the Creative Commons Attribution license (http://creativecommons.org/licenses/by/3.0/). 